\title{
On the Comparison of Stochastic Model Predictive Control Strategies Applied to a Hydrogen-based Microgrid
}

\author{
P. Velarde ${ }^{\mathrm{a}, 1}$, L. Valverde ${ }^{\mathrm{b}}$, J. M. Maestre ${ }^{\mathrm{a}}$, C. Ocampo-Martinez ${ }^{\mathrm{c}}$, C. Bordons ${ }^{\mathrm{a}}$ \\ ${ }^{a}$ System Engineering and Automation Department, School of Engineering, Universidad de Sevilla, 41092 \\ Seville, Spain. \\ ${ }^{b}$ AICIA, School of Engineering, University of Seville, 41092 Seville, Spain. \\ ${ }^{c}$ Automatic Control Department, Universidad Politécnica de Catalunya, Institut de Robòtica i Informàtica \\ Industrial (CSIC-UPC), 08028 Barcelona, Spain.
}

\begin{abstract}
In this paper, a performance comparison among three well-known stochastic model predictive control approaches, namely, multi-scenario, tree-based, and chance-constrained model predictive control is presented. To this end, three predictive controllers have been designed and implemented in a real renewable-hydrogen-based microgrid. The experimental set-up includes a PEM electrolyzer, lead-acid batteries, and a PEM fuel cell as main equipment. The real experimental results show significant differences from the plant components, mainly in terms of use of energy, for each implemented technique. Effectiveness, performance, advantages, and disadvantages of these techniques are extensively discussed and analyzed to give some valid criteria when selecting an appropriate stochastic predictive controller.
\end{abstract}

Keywords: Hydrogen storage, Microgrid, Model predictive control, Stochastic processes, Supply and demand.

\section{Introduction}

A microgrid is a network of electric generation that may take advantage of several renewable energy sources: solar panels, wind mini-generators, micro-turbines, fuel cells, among others, to meet the consumer demand by working together with the centralized grid or autonomously [1]. In a microgrid, the energy is generated only at certain times, being necessary to provide continuous service to meet the demand at any time of the day. Challenges arise from the natural intermittency of renewable energy sources and the requirements to satisfy the user energy demand [2]. Thereby, storage

Email addresses: pabvelrue@us.es (P. Velarde), lvalverde@etsi.us.es (L. Valverde), pepemaestre@us.es (J. M. Maestre), cocampo@iri.upc.edu (C. Ocampo-Martinez), bordonseus.es (C. Bordons)

${ }^{1}$ Corresponding author 


\begin{tabular}{|llll|}
\hline Nomenclature & & & \\
Symbols & & $e z$ & Electrolyzer \\
$E$ & Energy (Wh) & $f c$ & Fuel cells \\
$\mathbb{E}$ & Expected value & $g r i d$ & Grid \\
$F$ & Cumulative distribution function & $H_{2}$ & Hydrogen \\
$J$ & Cost function & $n e t$ & Net power \\
$K$ & Number of scenarios & res & Renewable energy source \\
$M H L$ & Metal hydrides level (\%) & ref & Reference \\
$N$ & Prediction horizon & Superscripts & \\
$P$ & Power (W) & $*$ & Working point \\
$\mathbb{P}$ & Probability & - & Measured value \\
$R$ & Reduced number of scenarios & Acronyms & \\
$S O C$ & State of charge $(\%)$ & ARMA & Autoregressive-moving-average \\
$a_{i}$ & Weight for state variables & CC & Chance-constrained \\
$b_{i}$ & Weight for input variables & cdf & cumulative distributed function \\
$u$ & Input variables & IID & Independent and identically distributed \\
$x$ & State variables & KPIs & Key performance indicators \\
$\omega$ & Disturbances & MPC & Model predictive control \\
$\Delta P$ & Rate power (Ws ${ }^{-1}$ ) & MS & Multiple-scenarios \\
$\delta_{x}$ & Risk of constraint violation & PEM & Proton exchange membrane \\
Subscripts & & PF & Perfect forecast \\
$b a t t$ & Batteries & SNEN & Spanish national electricity network \\
$d e m$ & Demand & UPG & Utility power grid \\
& & TB & Tree-based \\
\hline
\end{tabular}

devices become very important in the operation of this type of systems. Among wellestablished energy storage technologies, there are batteries, super-capacitors, conventional capacitors, etc. In this work, we focus on the use of hydrogen as an energy vector for energy storage. Hydrogen, combined with other renewable energy sources, is a safe and viable option to mitigate the problems associated with hydrocarbon combustion because the entire system can be developed as an efficient, clean, and sustainable energy source, as mentioned in [3]. The hydrogen is converted into electrical energy by using fuel cells; the reverse process, i.e., the transformation of electric energy into hydrogen, is conducted by electrolysis [4], or ethanol reforming [5], among other techniques.

The control problem in a microgrid is to satisfy the electricity demand under economical and optimal conditions despite the uncertainties and disturbances that might appear in the processes. Taking into account that there are mathematical models available that represent the main dynamics and the load of these systems [6], and that the control problem here requires the simultaneous handling of constraints, delays, and disturbances, model predictive control (MPC) emerges as a solution to this problem. MPC is a control strategy widely used in industry for solving problems considering constraints on the manipulated and controlled variables, delays, nonlinearities, etc. Very succinctly, the main idea of MPC is to obtain a control signal by solving at each time instant an optimization problem in a finite prediction horizon based on the system model. Only the first component of the control signal is implemented in the current time step, and the problem is solved in the next time instant in a receding horizon strategy. Due to its versatility, MPC has become one of the most popular techniques in industrial control applications; see e.g. [7]. From using the MPC approach, different variants of this technique have been applied to achieve an economical and optimal efficiency in energy management of a microgrid, see, e.g., [2, 8-13]. The potential benefits of 
the application of an MPC controller are discussed to solve this control problem in an economical manner. A review of the approaches applied for controlling smart grids have been presented in [14, 15] and references therein.

Uncertainty in the load and generation profiles has been mainly addressed indirectly in the dispatch problem by using the MPC approach [14]. The classical formulation of MPC does not allow considering systems with uncertainties although some MPC schemes have been proposed to ensure stability and compliance with constraints in the presence of disturbances [16]. One way to address this problem is by means of the conservative min-max approach, e.g., in [17] control of microgrids using this technique is shown. A less conservative approach is the stochastic one, which is based on the design of predictive controllers for dynamical systems subject to disturbances and/or uncertainty in terms of the probability that a certain solution is feasible [18], mainly because it is not strictly possible to speak about guaranteed feasibility in this context.

Due to the increasing importance that uncertainty plays in the power dispatch of smartgrids, stochastic MPC can be used to deal with the uncertainty in the energy demand and the renewable generation. At this point, the question of which method provides the best performance to manage the inherent uncertainties in microgrids arises. Although each method provides a different solution to the same problem, a comparison between the available techniques has been missed in the literature up to date. This is indeed the main contribution of this paper. Three popular stochastic MPC techniques have been implemented and tested to obtain experimental evidence in a real comparison framework of their suitability. The criteria to assess the controller performance is based on specific key performance indicators (KPIs) defined in this work. Also, guidelines for controller design, tuning, and real-time implementation in a hydrogen-based microgrid are also provided. Finally, the experimental results obtained highlight advantages and weaknesses when coping with disturbances and uncertainties within the closed loop of a hydrogen-based microgrid.

Therefore, in this paper, three different stochastic-programming-based MPC techniques are used to deal with the uncertainty of the power demand and power generation. In the first place, multiple-scenario MPC (MS-MPC) is considered, which consists in calculating a single control sequence that takes into account different possible evolutions of the process disturbances. Hence, the control sequence calculated has a certain degree of robustness against the potential realizations of the uncertainties. This approach is used for example in [19] for water systems and in [20, 21] within the context of the control of smart grids. One of its advantages is that it is possible to calculate bounds on the probability of constraint violation as a function of the number of scenarios considered [22].

An alternative to model the uncertainty that is faced by this type of systems is to use rooted trees. The rationale behind this approach is that uncertainty spreads with time, i.e., it is possible to predict -more accurately- both the energy demand and energy production by a renewable source in a short horizon than in a large one. For this reason, the possible evolutions of the disturbances can be confined to a tree. In the tree, there is a bifurcation point whenever the disturbances branch into two possible trajectories. Consequently, the outcome, the so-called tree-based MPC (TB-MPC), is a rooted tree of control actions. This approach is used for example in [23] for a semi-batch reactor example, in [24] for the energy management of a renewable hydrogen-based microgrid, 
and in [25] in the context of water systems.

Finally, chance-constrained MPC (CC-MPC) is also considered in this work. CCMPC uses an explicit probabilistic modeling of the system disturbances to calculate explicit bounds on the system constraint satisfaction. For instance, [26] presents a chanceconstrained two-stage stochastic program for unit commitment with uncertain wind power output and [27] shows an autoregressive-moving-average (ARMA) type prediction model for the underlying uncertainties (load/generation) into chance-constrained finite-horizon optimal control. An application of this technique in the context of the drinking water network of the city of Barcelona is reported in [28]. In addition, [29] shows a comparison between MS-MPC, TB-MPC, and CC-MPC approaches applied to drinking water networks via simulation. Further, this subject has drawn significant interest; a stochastic optimization model implemented in the context of the control of microgrids can be seen in [30-33] and references therein.

The remainder of the paper is organized as follows. First, a description of the microgrid, its linear model and constraints are shown in Section 2 Section 3 presents the optimization problem and the formulation of the considered stochastic MPC techniques. The comparative study and the analysis drawn from the experimental results are presented in Section 4. Finally, in Section 5 the main conclusions and future research lines are proposed.

\section{Case Study Description}

The microgrid under study is the lab-scale microgrid called HyLab [34]. The microgrid test bench used in this study is an experimental platform specifically designed for testing control strategies. HyLab is composed of a modular system equipped with various components that allow experimentation and simulation of several types of renewable energy sources. In the Figure 1, a picture of the experimental Hylab platform is shown.

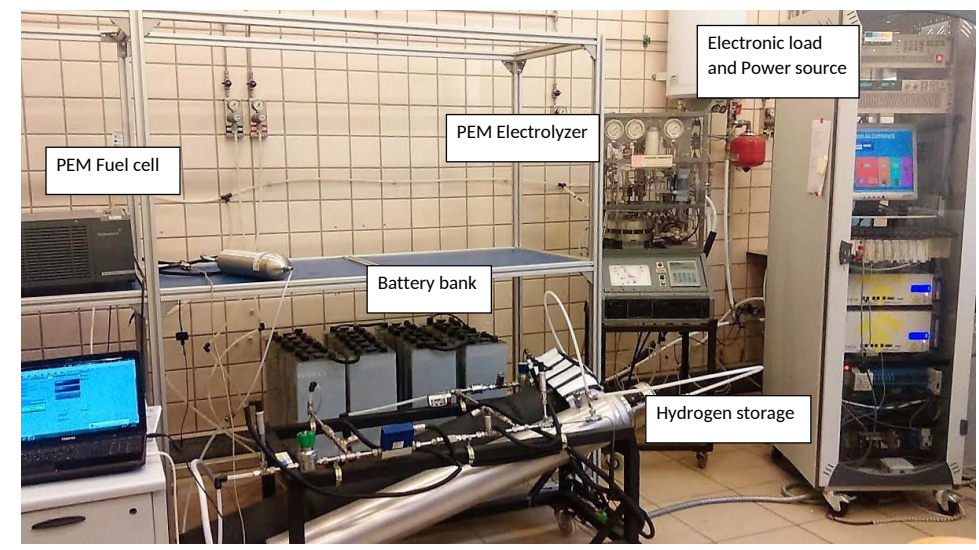

Figure 1: Experimental HyLab Plant. 
The system consists of a solar field, emulated by an electronic power source, which produces electricity to supply the electronic load. Any excess of power can be either stored in a battery bank or derived to the electrolyzer. If the power obtained from renewable energy is not enough, both the fuel cell and the battery bank can support the load, which is emulated electronically. This type of hybrid storage operation allows implementing strategies in separated times scales: the battery can either absorb or contribute to balance small amounts of energy in fast transient periods while the hydrogen path complements larger variations [35]. The microgrid can work either connected to the utility network or as an isolated system. The Hydrogen Path is composed of three subsystems: the electrolyzer, which is proton exchange membrane (PEM) type [36], for producing hydrogen; a metal-hydride hydrogen storage tank; and finally a PEM fuel cell [37, 38] that provides power to the loads/batteries. It is important to notice that both subsystems -electrolyzer and fuel cell-cannot work simultaneously. DC/DC power converters are used as power interfaces that allow energy transfer between different distributed generation units. The equipment is connected to $48 V_{\mathrm{DC}}$ bus that is held by the battery bank. Table 1 presents the nominal values of the HyLab equipment.

Table 1: HyLab equipment.

\begin{tabular}{ll}
\hline Equipment & Nominal Value \\
\hline \hline & \\
Electronic power source & $6 \mathrm{~kW}$ \\
Electronic load source & $2.5 \mathrm{~kW}$ \\
PEM fuel cell & $1.2 \mathrm{~kW}$ \\
PEM electrolyzer & $0.23 \mathrm{Nm}^{3} \mathrm{~h}^{-1} @ 5 \mathrm{barg}$ \\
& $1 \mathrm{~kW}$ \\
Metal hydrides tank & $7 \mathrm{Nm}^{3}$ \\
& $5 \mathrm{bar}$ \\
Battery bank & $C_{120}=367 \mathrm{Ah}$ \\
DC/DC converters & $1.5 \mathrm{~kW}, 1 \mathrm{~kW}$ \\
\hline
\end{tabular}

\subsection{Microgrid linear model and constraints}

As it can be inferred, behind the experimental setup there is a set of complex nonlinear subsystems. The detailed description of sub-models and the physical equations are out of the scope of this paper. The complete non-linear model of the plant, its simulation, and validation are presented in [39].

Remark 1. To apply linear MPC techniques is required to find a linear model of the system around a working point $\left(x^{*}, u^{*}\right)$. The identification process for obtaining the linear model of the plant is developed in [9]. The continuous linear system was discretized using Tustin's method with a sampling time of $30 \mathrm{~s}$. Also, the working point is given by $u^{*}=[0 \mathrm{~kW}, 1.75 \mathrm{~kW}]^{T}$ and $x^{*}=[50 \%, 50 \%]^{T}$. 
The linear discrete-time model of the plant consists of two input variables, $P_{\mathrm{H}_{2}}(k)$ and $P_{\text {grid }}(k)$, which are measured in kilowatts $(\mathrm{kW})$. Here, $P_{\mathrm{H}_{2}}(k)$ represents the power of the electrolyzer and the power of the fuel cell: when it is greater than zero, the PEM fuel cell is working $\left(P_{\mathrm{fc}}(k)\right)$, and when $P_{\mathrm{H}_{2}}(k)$ is negative, it indicates that the electrolyzer is operating $\left(P_{\mathrm{ez}}(k)\right)$. Both the electronic load and the electronic power source can either deliver or absorb power from the utility power grid (UPG). The connection with the electric network is "virtual", since it is emulated by the source and electronic load. Moreover, $P_{\text {grid }}(k)$ represents the power of UPG, which is positive when the power is imported by the microgrid from the UPG, and it is negative when exporting power to the UPG. The system is subject to uncertainties from the power produced by a renewable energy source, in this case, it is the power from the solar field, $\left(P_{\text {res }}(k)\right)$ and the power demanded by the consumers $\left(P_{\text {dem }}(k)\right)$; the difference between them can be considered as disturbances $\left(P_{\text {net }}(k)\right)$ to the system. Moreover, the plant counts with an additional variable, the power of the batteries $\left(P_{\text {batt }}\right)$, which is controlled indirectly, resulting of the power balance. The states are given by the state of charge of the batteries $(S O C(k))$, and the metal hydrides level $(M H L(k))$ of the storage tank, both measured in percentage (\%). A scheme of the power variables is shown in Figure 2. The discrete-time linear model of the plant, for each time instant $k \in \mathbb{Z}_{+}$, around a working point $\left(u^{*}, x^{*}\right)$, can be written as

$$
x(k+1)=A x(k)+B u(k)+D \omega(k),
$$

that is,

$$
x(k+1)=x(k)+\left[\begin{array}{cc}
8.1360 & 5.958 \\
-15.2886 & 0
\end{array}\right] u(k)+\left[\begin{array}{c}
5.958 \\
0
\end{array}\right] \omega(k) .
$$

In this model, $u(k)=\left[P_{\mathrm{H} 2}(k), P_{\text {grid }}(k)\right]^{T}$ represents the vector of manipulated variables, $x(k)=[S O C(k), M H L(k)]^{T}$ is the state vector of the system and $\omega(k)=$ $P_{\text {net }}(k) \in \mathbb{R}^{n_{d}}$ represents the system disturbance, where $n_{d}=1$.

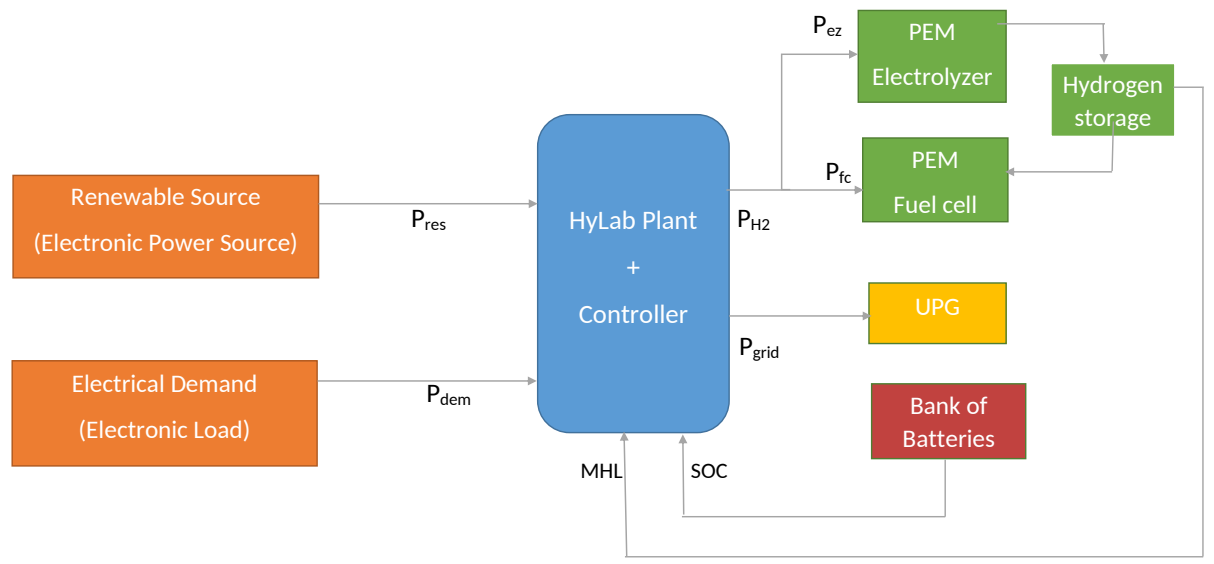

Figure 2: HyLab variables scheme. 
The system is subject to constraints that avoid equipment damage and guarantee its safe operation. In particular, the Hydrogen Path -both the electrolyzer and the fuel cellhas constraints for limiting the values of $P_{\mathrm{H}_{2}}(k)$ since its power capacity is limited to $0.9 \mathrm{~kW}$; this value reflects some conservatism and it ensures that the hydrogen path does not work at its nominal value to protect the equipment. In this way, a longer lifespan is expected. Also, the Hydrogen Path has a dead zone between $-0.1 \mathrm{~kW}$ and $0.1 \mathrm{~kW}$ that ensures a minimum production of power from both the electrolyzer and the fuel cell. The constraints for $P_{\text {grid }}(k)$ correspond to physical limitations of the electronic units. Furthermore, it is necessary to include constraints on their incremental signals $\Delta P_{\mathrm{H}_{2}}(k)$ and $\Delta P_{\text {grid }}(k)$, to guarantee the physical safety of the equipment. These constraints are mathematically expressed as follows:

$$
\begin{aligned}
& -0.9 \mathrm{~kW} \leq P_{\mathrm{H}_{2}}(k) \leq 0.9 \mathrm{~kW}, \\
& -2.5 \mathrm{~kW} \leq P_{\text {grid }}(k) \leq 2 \mathrm{~kW}, \\
& -20 \mathrm{Ws}^{-1} \leq \Delta P_{\mathrm{H}_{2}}(k) \leq 20 \mathrm{Ws}^{-1}, \\
& -2.5 \mathrm{kWs}^{-1} \leq \Delta P_{\text {grid }}(k) \leq 2 \mathrm{kWs}^{-1} .
\end{aligned}
$$

Overall constraints have to be considered as hard constraints, since the equipment lifespan could be drastically reduced. Both the battery bank and the metal hydrides storage tank have limited capacity to prevent any plant damage by overcharge or undercharge. Constraints on $S O C(k)$ guarantee suitable voltage levels in the $48 V_{D C}$ bus. Also, they protect the battery bank of strong load voltage variations. These state constraints are written as

$$
\begin{aligned}
& 40 \% \leq S O C(k) \leq 90 \% \\
& 10 \% \leq M H L(k) \leq 90 \% .
\end{aligned}
$$

The input constraints given by (2) can be properly rewritten as

$$
u(k) \in \mathcal{U} \subseteq \mathbb{R}^{n_{u}},
$$

with $n_{u}=2$, while the state constraints defined by (3) are expressed as

$$
x(k) \in \mathcal{X} \subseteq \mathbb{R}^{n_{x}},
$$

with $n_{x}=2$. Furthermore, the total power delivered to the load, in order to satisfy the consumer demand, must satisfy the energy balance

$$
P_{\text {dem }}(k)=P_{\mathrm{H} 2}(k)-P_{\text {batt }}(k)+P_{\text {grid }}(k)+P_{\text {res }}(k) .
$$

\section{Stochastic MPC formulation for hydrogen-based microgrids}

MPC is a strategy based on the explicit use of a dynamical model of the plant to predict the state/output evolution of the process in future time instants along a prediction horizon $N$ [7]. The set of future control signals is calculated by the optimization of 
a criterion or objective function. Only the control signal calculated for the time instant $k$ is applied to the process, whereas the others are withdrawn. One of the advantages of MPC over other control methods includes the easy extension to the multivariable case.

The optimization problem to be solved at each time instant $k$ is formulated as

$$
\min _{\{u(k), \ldots, u(k+N-1)\}} \sum_{i=0}^{N-1} J(x(k+i), u(k+i)),
$$

subject to

$$
\begin{aligned}
& x(i+1)=A x(i)+B u(i)+D \omega(i), \\
& x(0)=x(k), \\
& x(i+1) \in \mathcal{X}, \\
& u(i) \in \mathcal{U}, \quad \forall i \in \mathbb{Z}_{0}^{N-1},
\end{aligned}
$$

where $A \in \mathbb{R}^{n_{x} \times n_{x}}, B \in \mathbb{R}^{n_{x} \times n_{u}}$, and $D \in \mathbb{R}^{n_{x} \times n_{d}}$ are the system matrices defined in (1). The multi-objective cost function to be minimized is given by

$$
\begin{aligned}
J(x(k), u(k)) & =a_{1}\left(S O C(k)-S O C_{\mathrm{ref}}\right)^{2} \\
& +a_{2}\left(M H L(k)-M H L_{\mathrm{ref}}\right)^{2} \\
& +b_{1} P_{\mathrm{H}_{2}}^{2}(k)+b_{2} P_{\text {grid }}^{2}(k) .
\end{aligned}
$$

The sequence of inputs that must be applied to the plant along the horizon is denoted by $\{u(k), \ldots, u(k+N-1)\}$. Note that only $u(k)$ is actually applied. Here, $S O C_{\mathrm{ref}}=65 \%$ and $M H L_{\mathrm{ref}}=40 \%$ are the references given for the state of charge of the batteries and the metal hydride level, respectively. The tuning of the cost function weights seeks for a soft tracking of the output variables towards the given references and an efficient use of the energy. More specifically, the controller is designed such that the batteries are the first way of energy storage. If there exists a big difference between the demanded energy and the produced energy by the renewable sources, it proceeds to the production of hydrogen. These prioritization weights $a_{i}, b_{i}$ have been adjusted by trial and error approach carried out on simulation tests reported in previous works with this plant, see, e.g. [4, 8, 9]. In this sense, they have been established as $a_{1}=a_{2}=10, b_{1}=5000$, and $b_{2}=8000$. As can be seen, the weight associated with the hydrogen production is lower than the weight related to the power of the grid in order to minimize the power interchange with the UPG. The weights associated with the outputs take low values compared with the others to give flexibility to the smart grid. However, these values can been modified in the multi-objective function (9) for tracking the reference. In this work, the energy management is the main objective, therefore the weights associated with the hydrogen path and the grid are higher than those associated with the outputs of the system.

In this paper, MPC is used to satisfy the power demanded by local consumers. As mentioned before, both the demand and the generation of energy show a stochastic behavior. Therefore, the use of stochastic MPC techniques that account for the uncertainty is well suited for this context. 
Next, the description of the stochastic MPC techniques designed and implemented is presented.

\subsection{Multiple-scenarios MPC approach (MS-MPC)}

The optimization based on scenarios provides an intuitive way to approximate the solution to the stochastic optimization problem. In order to design the MS-MPC, it is required to know several scenarios with possible evolutions of the energy demand and generation. The scenario forecasts can be obtained either from historical data or by introducing a random scenario generation. The idea behind this approach is that a general control sequence that optimizes all the considered scenarios is calculated, obtaining in this way a certain robustness against the different possible evolutions of the disturbances. The scenario-based approach is computationally efficient since its solution is based on a deterministic convex optimization, even when the original problem is not [40]. One advantage of this approach does not assume a prior knowledge of the statistical properties that characterize the uncertainty (e.g., a certain probability function) as generally required in stochastic optimization.

The main idea for optimization with a finite number of scenarios is to consider the same system for each one of the known disturbance realizations. The problem consists in solving

$$
\min _{\{u(k), \ldots, u(k+N-1)\}} \sum_{j=1}^{K}\left(\sum_{i=0}^{N-1} J\left(x_{j}(k+i), u(k+i)\right)\right),
$$

subject to

$$
\begin{aligned}
& x_{j}(i+1)=A x_{j}(i)+B u(i)+D \omega_{j}(i), \\
& x_{j}(0)=x(k), \\
& \omega_{j}(i)=\widehat{\omega}_{j}(i), \\
& x_{j}(i+1) \in \mathcal{X}, \\
& u(i) \in \mathcal{U} \quad \forall i \in \mathbb{Z}_{0}^{N-1}, \quad \forall j \in \mathbb{Z}_{1}^{K},
\end{aligned}
$$

where $K \in \mathbb{Z}_{+}$is the finite number of scenarios considered and $\widehat{\omega}_{j}(k)$ is the disturbance forecast for scenario $j \in \mathbb{Z}_{1}^{K}$.

Due to the stochastic nature of the disturbances, the number of scenarios considered $K$ deserves special attention to ensure compliance with the state constraints with a certain confidence degree, i.e.,

$$
\mathbb{P}\left[x_{j}(i+1) \in \mathcal{X}\right]>1-\delta_{x},
$$

where $\mathbb{P}[\cdot]$ denotes the probability operator and $\delta_{x} \in(0,1)$ is the risk acceptability level of constraint violation for the states. The number of scenarios needed to achieve this goal can be calculated as a function of $\delta_{x}$, the number of variables in the optimization problem $(z)$, and a quite small confidence level $\left(\beta \leq 10^{-6}\right)$, as indicated in [41]

$$
K \geq \frac{z+1+\ln \left(\frac{1}{\beta}\right)+\sqrt{2(z+1) \ln \left(\frac{1}{\beta}\right)}}{\delta_{x}} .
$$


Furthermore, the sample scenarios must meet the following assumptions, as pointed out in [40]:

1. The uncertainties $\widehat{\omega}_{j} ; \forall j \in \mathbb{Z}_{1}^{K}$ are independent and identically distributed (IID) random variables on a probability space.

2. A "sufficient number" of IID samples of $\widehat{\omega}_{j}$ can be obtained, either empirically or by a random-number generator.

In this manner, a control sequence is optimized for the system given by 11a, which includes different possible evolutions of the original one. The calculation of the controller will result in a unique robust control action that satisfies all the potential realizations of the disturbances with a certain probability.

\subsection{Tree-based $M P C(T B-M P C)$}

This technique consists of transforming the different possible evolutions of the disturbances into a rooted tree that, through its evolution, diverges and generates a reduced number of scenarios. The points of divergence are called bifurcations and they represent moments in time in which the potential evolution of the disturbances is uncertain enough to consider more than one trajectory, as shown in Figure 3 . The formulation of the control problem involves making tree-based optimization scenarios, where only the most relevant disturbance patterns are modeled, starting with a common root that corresponds to the current disturbance at each time instant. It must be noted that TB-MPC formulates the optimization problem by means of Multistage Stochastic Programming [42, 43]. The number of scenarios used to build the tree should be coherent with the computational capability of the controller and the risk probability, $\delta_{x}$.
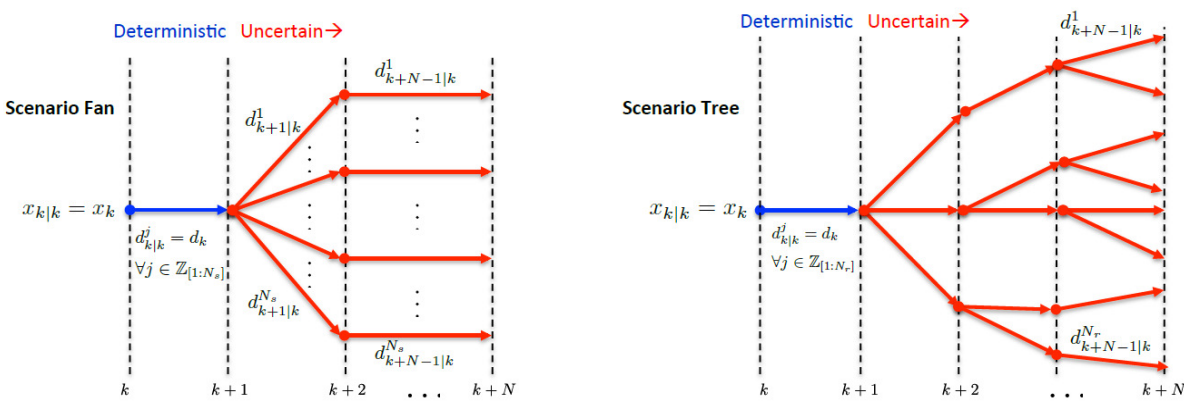

Figure 3: Scenario fan and scenario tree over the prediction horizon.

Being a scenario-based approach, it is possible to determine $\delta_{x}$ by taking into account the number of discarded scenarios $N_{r}$ from the initial $K$ scenarios for any violation level $v \in[0,1]$, as seen in [40]. The probability of satisfying the state constraints is given by

$$
\mathbb{P}\left[x_{i+1} \in \mathcal{X}\right] \geq 1-\delta_{x}
$$

where

$$
\delta_{x}=\int_{0}^{1} U(v) d v
$$


and

$$
U(v)=\min \left\{1,\left(\begin{array}{c}
N_{r}+z-1 \\
N_{r}
\end{array}\right) \sum_{j=0}^{N_{r}+z-1}\left(\begin{array}{c}
K \\
j
\end{array}\right) v^{j}(1-v)^{K-j}\right\} .
$$

In this way, the amount of $R$ used in the optimization problem is calculated as $R=K-N_{r}$.

Unlike the MS-MPC approach, each scenario into the tree has its own control signal, which means that more optimization variables are needed. However, given that the control signal cannot anticipate events beyond the next bifurcation point, control sequences for different scenarios must be equal as long as the scenarios do not branch out. As a consequence, the solution of this control problem is a rooted-tree of control inputs. Notice that only the first component of this tree, which is equal for all the scenarios, is actually applied. For the design of this controller, the bifurcation points of the tree are checked: if they are equal, then the control actions are the same so that both the number of variables and the computational time can be reduced significantly.

The TB-MPC problem formulation to be solved at each time instant is represented by

$$
\min _{\left\{u_{j}(k), \ldots, u_{j}(k+N-1)\right\}} \sum_{j=1}^{R}\left(\sum_{i=0}^{N-1} J\left(x_{j}(k+i), u_{j}(k+i)\right)\right),
$$

subject to

$$
\begin{aligned}
& x_{j}(i+1)=A x_{j}(i)+B u_{j}(i)+D \omega_{j}(i), \\
& x_{j}(0)=x(k), \\
& \omega_{j}(i)=\widehat{\omega}_{j}(i), \\
& x_{j}(i+1) \in \mathcal{X}, \quad \forall i \in \mathbb{Z}_{0}^{N-1}, \\
& u_{j}(i) \in \mathcal{U}, \quad \forall j \in \mathbb{Z}_{1}^{R} .
\end{aligned}
$$

In addition, it is necessary to introduce non-anticipative constraints to force the controller to compute the control inputs only considering the observed uncertainty before the bifurcation points [43]. These constraints are given by

$$
u_{i}(k)=u_{j}(k) \quad \text { if } \quad \widehat{\omega}_{i}(k)=\widehat{\omega}_{j}(k) ; \forall i \neq j .
$$

One way to satisfy (15f] is to introduce equality constraints into the optimization problem and solving it with a number of optimization variables defined as $z=N \times R \times$ $n_{u}$. Nevertheless, constraints in (15f) can be used to reduce the number of optimization variables by removing the redundancy to lower the computational burden.

As said before, a control sequence is optimized for the extended system with a disturbance tree, and only the first component of the input tree is applied to the system. The problem is repeated at each time instant $k \in \mathbb{Z}_{+}$. 


\subsection{Chance-Constrained MPC (CC-MPC)}

Given that disturbances are stochastic, another way of addressing this problem is using CC-MPC. The stochastic behavior from the weather conditions and the electric demand can be addressed by formulating hard constraints into probabilistic constraints related to a risk of constraint violation that determines the degree of the conservatism when computing the control inputs. Also, the cost function is expressed as its expected value in the formulation of the optimization problem. A major advantage of this approach is that the computational burden is not increased as in the scenario-based techniques.

Given that the disturbances in the dynamic model (1a) are stochastic, the state constraints (5) must be formulated in a probabilistic manner, i.e.,

$$
\mathbb{P}[x(i+1) \in \mathcal{X} \mid G x \leq g]>1-\delta_{x} .
$$

Here, $G \in \mathbb{R}^{n_{r} \times n_{x}}$ and $g \in \mathbb{R}^{n_{r}}$. The probabilistic constraints $(16)$, also called chance constraints, can be written in two different manners [28]:

- Individual chance constraints that express a probabilistic equivalent for each constraint. They are formulated as

$$
\mathbb{P}\left[G_{(m)} x<g_{(m)}\right]>1-\delta_{x, m}, \quad \forall m \in \mathbb{Z}_{1}^{n_{x}},
$$

where $G_{(m)}$ and $g_{(m)}$ are the $m^{\text {th }}$ row of $G$ and $g$, respectively. Each $m^{\text {th }}$ row satisfies its respective $\delta_{x, m}$.

- Joint chance constraints, which take into account an unique risk of constraint violation for all stochastic constraints. They are written as

$$
\mathbb{P}\left[G_{(m)} x<g_{(m)}, \quad \forall m \in \mathbb{Z}_{1}^{n_{x}}\right]>1-\delta_{x} .
$$

All rows jointly satisfy the unique $\delta_{x}$.

The application of $(18)$ along $N$ is necessary to implement the controller. To this end, it is assumed that the disturbances behave as Gaussian random variables, which are modeled based on historical data, with a known cumulative distribution function (cdf). The deterministic equivalent of these chance constraints can be formulated as follows:

$$
\begin{aligned}
\mathbb{P}\left[G_{(m)} x(k+1)\right. & \left.<g_{(m)}\right]>1-\delta_{x} \\
& \Leftrightarrow F_{G_{(m)} D \omega(k)}\left(g_{(m)}-G_{(m)}(A x(k)+B u(k))\right)>1-\delta_{x} \\
& \Leftrightarrow G_{(m)}(A x(k)+B u(k))<g_{(m)}-F_{G_{(m)} D \omega(k)}^{-1}\left(1-\delta_{x}\right) .
\end{aligned}
$$

Here, $F_{G_{(m)}} D \omega(k)(\cdot)$ represents the cumulative distribution function of the random variable $G_{(m)} D \omega(k)$, and $F_{G_{(m)} D \omega(k)}^{-1}(\cdot)$ is its inverse cumulative distribution function.

Note that the expression (19) is the deterministic equivalent of the chance constraints and is built based on historical data. 
The optimization problem formulation related to the design of the CC-MPC controller is stated as

$$
\min _{\{u(k), \ldots, u(k+N-1)\}} \sum_{i=0}^{N-1} \mathbb{E}[J(x(k+i), u(k+i))],
$$

subject to

$$
\begin{aligned}
& x(i+1)=A x(i)+B u(i)+D \omega(i), \\
& x(0)=x(k), \\
& \omega(i)=\widehat{\omega}(i), \\
& G_{(m)}(A x(k)+B u(k))<g_{(m)}-F_{G_{(m)} D \omega(k)}^{-1}\left(1-\delta_{x}\right), \\
& u(i) \in \mathcal{U}, \quad \forall i \in \mathbb{Z}_{1}^{N-1},
\end{aligned}
$$

where $\mathbb{E}[\cdot]$ denotes the expected value of the cost function.

\section{Results and Discussion}

The experiments were conducted in the microgrid described in Section 2 during a trial period of eight hours for each experiment. The controller receives the measured variables $S O C(k)$ and $M H L(k)$, which are used to compute the optimal control signals $P_{\mathrm{H} 2}(k)$ and $\left.P_{\text {grid }}(k)\right)$ by means of Simulink Real-Time workshop toolbox. The control signals are sent to the SCADA via the OPC Matlab Library and finally the PLC carries out these control actions.

The prediction horizon was $N=5$ and the sampling time was $30 \mathrm{~s}$. The selected weather and load profiles for verifying the performance of the three proposed controllers were the scaled difference between the real solar generation and the demand registered by the Spanish National Electricity Network (SNEN) ${ }^{2}$ on May 23, 2014. These values were sampled each $3 \mathrm{~s}$ and scaled for the microgrid allowable power values, which are shown in Figure 4 (a). The initial conditions for all experiments were $S O C(0)=70 \%$ and $M H L(0)=50 \%$.

An issue that deserves particular attention is the amount of scenarios to be considered into the optimization problem. This number should be selected by taking into account a trade-off between robustness and computational burden. In this sense, it is possible to establish the number of scenarios that guarantees a particular risk level, according to (12), as shown Table 2 .

MS-MPC was performed by using the electricity demand and the solar generation registered during $K=316$ different days of one year from historical data, obtained from the SNEN. For these scenarios, it is expected a risk of violation of constraints less than $\delta_{x} \leq 10 \%$. This number of scenarios offers an acceptable risk and ensures a reasonable computational burden when solving the optimization problem. Furthermore,

${ }^{2}$ SNEN demand data can be obtained at: https://demanda.ree.es/movil/peninsula/demanda/total 
Table 2: Number of scenarios $(\mathrm{K})$ that fulfills an specific risk level $\left(\delta_{x}\right)$.

\begin{tabular}{|l|l|l|l|l|l|}
\hline$\delta_{x}$ & 0.20 & 0.15 & 0.10 & 0.05 & 0.01 \\
\hline$K$ & 152 & 203 & 316 & 611 & 3005 \\
\hline
\end{tabular}

this set of scenarios considers days with enough solar energy generation as well as cloudy days, which makes the controllers more robust and somehow relieves the need for increasing the number of scenarios used. TB-MPC was performed by using an original number of $K=316$ scenarios, which were reduced to $R=250$ scenarios forming a tree using GAMS [44]. This reduction tried to replicate the main dynamics of all original disturbances considered in a small disturbance tree. This reduction introduces a boundary that guarantees $\delta_{x} \leq 10 \%$, according to 13 . Finally, CC-MPC approach was performed considering the failure probability $\delta_{x}=10 \%$. The disturbances were considered as a random function with a cdf, which were obtained from the historical daily data registered in 2014 .

The scheme of the microgrid operation, from a general point of view, follows similar patterns for the three proposed controllers. At this point, given that the energy from the renewable source is not sufficient to meet the energy demand, the fuel cell turns on, the battery $S O C$ and the $M H L$ decrease gradually without going below their forbidden levels. Also, energy is imported from the grid to meet the load beyond demand. When the energy from the renewable source greatly exceeds demand, the electrolyzer is switched on, the batteries are fully charged, and the excess of energy is stored in the hydrogen tank, and the remaining power that cannot be stored in the form of hydrogen is exported to the grid. However, each stochastic MPC approach shows particular differences, as reported below, which are highlighted to offer a suitable comparison among them.

In order to compare all the considered strategies, Figure 4(b) shows the battery power for the three aforementioned stochastic predictive controllers. As can be noticed, CC-MPC controller performs a deep cycle using the batteries. It strives for using the full capacity, reaching the upper and lower levels. In contrast, TB-MPC controller, although partly discharges and recharges the batteries, it does in a softer way. This implies that the excess of energy must be balanced through either the electrolyzer or the grid. It is observed that MS-MPC technique behaves between the two other approaches. Therefore, MS-MPC controller achieves a trade-off between using the full capacity of the batteries and the energy derived either to the electrolyzer or the grid.

Figure 4(c) presents the fuel cell and electrolyzer power along the test duration. The fuel cell performance signals obtained are similar for all the three controllers, except for CC-MPC controller, which shows a peak between the first and second hour, to satisfy an increase in the energy demand at that time. When there is an excess of energy from the renewable source, the electrolyzer starts its operation. Results show a clear difference in the electrolyzer operation. On the one hand, with CC-MPC technique, the electrolyzer presents a larger use of the power, as expected. On the contrary, the electrolyzer utilization is restricted quite more with TB-MPC approach, reaching only a 


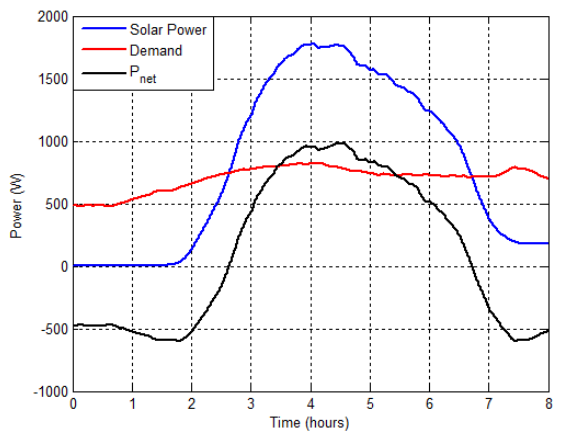

(a) Energy generated by solar panels $P_{\text {res, }}$ demand of energy $P_{\text {den, }}$ and $P_{\text {net }}$ corresponding to May $23,2014$.

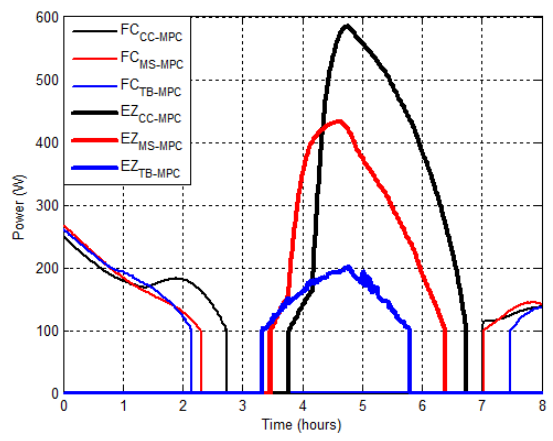

(c) Fuel cell power and Electrolyzer power.

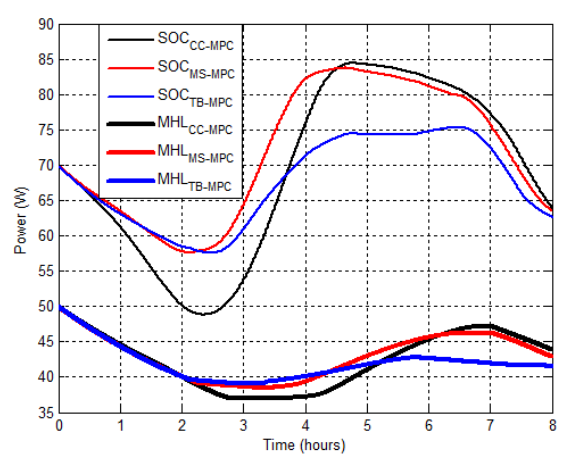

(e) Battery SOC and MHL

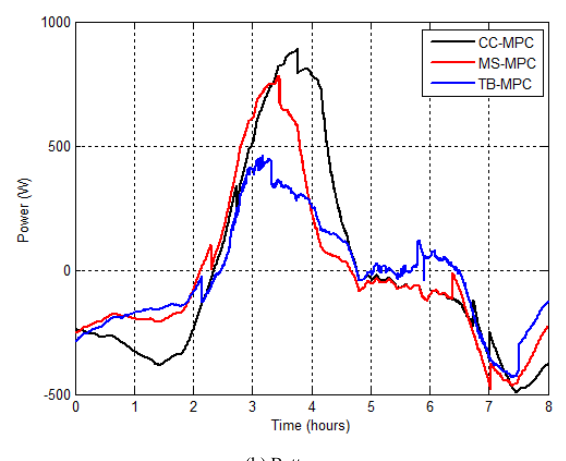

(b) Battery power.

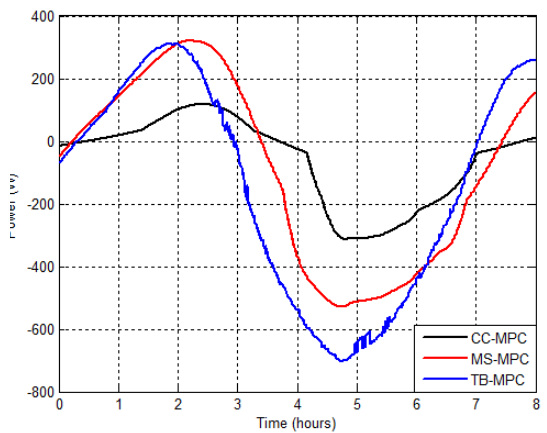

(d) Grid power.

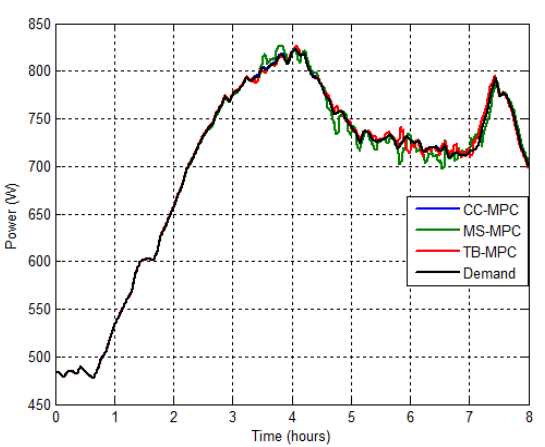

(f) Electric power provided by the microgrid compared with the consumer demand.

Figure 4: Experimental results applying the proposed stochastic MPC approaches.

peak of $200 \mathrm{~W}$, while CC-MPC controller sets the electrolyzer power to nearly $600 \mathrm{~W}$. Regarding TB-MPC approach, it also shows a small ripple; this is explained because the controller seeks to primary satisfy the demand and compensate any power unbal- 
ance in the system. As it has been shown through experimental tests, there are clear differences in the way each controller manages the power signals of the electrolyzer and the fuel cell.

Figure 4(d) shows the grid power signal generated by applying the stochastic MPC controllers. From the point of view of the network operators ( $\mathrm{DSO}^{3} \mathrm{TSO}^{4}$, the use of the UPG is minimized with the CC-MPC approach. In this manner, the impact in the electrical system generated by the renewable sources present in the microgrid is reduced. On the other hand, for the consumer point of view, it might be convenient not to force the equipment to a deep duty cycle and take advantage of the grid to smooth the power profiles.

Figure $4(\mathrm{e})$ shows the evolution of the $S O C$ and $M H L$ for each proposed controllers along the test period. In general, for all the implemented controllers, the batteries are discharged until the fuel cell turns off at the first time, and then they raise their charge level lower than $85 \%$ for MS-MPC and CC-MPC controllers. Regarding TB-MPC controller, it holds a charge level around $75 \%$ for a longer period compared with the other ones. Then, the $S O C$ starts to decrease again for all controllers under study. The $M H L$ presents a minor variation, and it reduces its level below $40 \%$ until the renewable source can contribute with power to the load. After this, the $M H L$ seeks to track its reference.

Figure 4 f) shows the comparison among the different powers delivered to the load by applying the controllers. As seen, the demand is satisfied by the power from the microgrid for all the controllers as imposed in their design. Notice that, in some situations, using the "elasticity" of the consumer; it might be possible to momentarily unbalance the power demand to satisfy other microgrid objectives [45]. Nevertheless, demand response is out of the scope of this paper.

In order to quantitatively assess the performances of these three stochastic approaches that have been implemented in the HyLab microgrid laboratory, several KPIs have been defined as follows:

- $\mathrm{KPI}_{1}$ defines the final cumulative cost given by (9) (in cost units).

- $\mathrm{KPI}_{2}$ is the computational time to solve the optimization problem (in s).

- $\mathrm{KPI}_{3}$ counts the average unmet demand with respect to the overall power demand (in \%).

- $\mathrm{KPI}_{4}$ is the time that the fuel cell is operating (in hours).

- $\mathrm{KPI}_{5}$ is the time that the electrolyzer is operating (in hours).

- $\mathrm{KPI}_{6}$ indicates the final value of $S O C$ (in \%).

- $\mathrm{KPI}_{7}$ indicates the final value of $M H L$ (in \%).

\footnotetext{
${ }^{3}$ DSO: Distributed System Operator

${ }^{4}$ TSO: Transmission System Operator
} 
Table 3 summarizes the numerical results of the KPIs. As it can be seen the highest value for $\mathrm{KPI}_{1}$ is obtained when using MS-MPC. The rationale behind this value is that the controller optimizes a sequence of control actions valid for the most favorable scenarios as well as the least favorable ones. In this sense, an over-conservative control action is carried out. This issue can be relaxed by calculating a tree of control actions that is subject to non-anticipatory equality constraints. In this way, the control actions are calculated in a closed-loop fashion, i.e., the controller can adapt the future control actions to the evolution of the disturbances. As can be seen, TB-MPC reduces its cumulative cost by increasing the number of control variables involved into the optimization problem. Hence, its computational time is the biggest within this comparative study. Regarding CC-MPC, it has the lowest cumulative cost without increasing the number of control variables. For reference purposes, the final cumulative cost for an MPC with a perfect forecast (PF-MPC), obtained via simulation, is $2.05 \times 10^{12}$. The computational time comparison is provided by $\mathrm{KPI}_{2}$.

Table 3: Comparison of the MS-MPC, TB-MPC, and CC-MPC controllers applied to HyLab microgrid by means of $\mathrm{KPI}_{i}, \quad i=1, \ldots, 7$.

\begin{tabular}{llllllll}
\hline Controller & $\begin{array}{l}\mathbf{K P I}_{1} \\
\text { (cost units) }\end{array}$ & $\begin{array}{l}\mathbf{K P I}_{2} \\
(\mathrm{~s})\end{array}$ & $\begin{array}{l}\mathbf{K P I}_{3} \\
(\%)\end{array}$ & $\begin{array}{l}\mathbf{K P I}_{4} \\
\text { (hours) }\end{array}$ & $\begin{array}{l}\mathbf{K P I}_{5} \\
\text { (hours) }\end{array}$ & $\begin{array}{l}\mathbf{K P I}_{6} \\
(\%)\end{array}$ & $\begin{array}{l}\mathbf{K P I}_{7} \\
(\%)\end{array}$ \\
\hline \hline MS-MPC & $3.89 \times 10^{12}$ & 7.76 & 0.12 & 3.26 & 2.90 & 63.53 & 42.91 \\
TB-MPC & $2.75 \times 10^{12}$ & 18.15 & 0.11 & 2.63 & 2.45 & 62.51 & 41.58 \\
CC-MPC & $2.44 \times 10^{12}$ & 1.04 & 0 & 3.70 & 2.97 & 63.71 & 43.85 \\
\hline
\end{tabular}

The three tested controllers are able to meet the overall demand in a satisfactory way, as indicated by the performance comparison given by $\mathrm{KPI}_{3}$. At this point, we must remark that the three tested controllers solve their optimization problems faster than the sampling time. Therefore, it is possible to select the approach that has the best performance in terms of the demand satisfaction and the use of the hydrogen path.

The comparisons between the proposed controllers regarding the time when both the fuel cell and the electrolyzer are operating are given by $\mathrm{KPI}_{4}$ and $\mathrm{KPI}_{5}$, respectively. In this sense, TB-MPC shows the lowest time for the fuel cell and the electrolyzer. It offers a larger conservatism when working with the hydrogen path, which is obtained at the expense of a higher computational time since TB-MPC meets the current demand and reformulates its disturbance tree at each time step. Notice that the main difference is at the time that the hydrogen path is working.

The final values of $S O C$ and $M H L$, which present similar values for the three controllers, are around $63 \%$ and $42 \%$, respectively. These values are provided by $\mathrm{KPI}_{6}$ and $\mathrm{KPI}_{7}$.

Table 4 presents a comparison among the total energy produced by the fuel cell $\left(E_{\mathrm{fc}}\right)$, the electrolyzer $\left(E_{\mathrm{ez}}\right)$, the batteries $\left(E_{\mathrm{batt}}\right)$, and the grid $\left(E_{\text {grid }}\right)$ during the test period. The negative sign in $E_{\text {grid }}$ indicates that the amount of energy sold to UPG is greater than the energy purchased. The total energy of the batteries indicates the difference between the stored energy and the delivered energy to the load: the negative 
value means that the stored energy predominates over the delivered energy.

Table 4: Energy produced by the fuel cell, electrolyzer, batteries, and grid during the test period by applying the proposed stochastic MPC controllers.

\begin{tabular}{lllll}
\hline Controller & $\begin{array}{l}E_{\mathrm{fc}} \\
(\mathrm{Wh})\end{array}$ & $\begin{array}{l}E_{\mathrm{ez}} \\
(\mathrm{Wh})\end{array}$ & $\begin{array}{l}E_{\mathrm{batt}} \\
(\mathrm{Wh})\end{array}$ & $\begin{array}{l}E_{\mathrm{grid}} \\
(\mathrm{Wh})\end{array}$ \\
\hline \hline MS-MPC & 302 & 481 & -62.2 & -418 \\
TB-MPC & 261 & 217 & -110.23 & -661 \\
CC-MPC & 348 & 642 & -43.09 & -268 \\
\hline
\end{tabular}

Notice that the absolute value of the energy amounts are taken to achieve a reliable comparison in terms of energy consumption for each component of the system. In this sense, CC-MPC has better performance regarding energy efficiency. CC-MPC achieves less exchange with UPG, and the batteries provide enough power to supply the load. Also, both the fuel cell and electrolyzer use energy in a wider range when compared to the MS-MPC and TB-MPC approaches. Note also that TB-MPC and MSMPC handled more cautiously hydrogen energy from the path while performing more exchanges with the UPG, specially TB-MPC.

Another KPI to compare the performance of the controllers for energy management in a smartgrid is the number of start-ups for both equipment, the fuel cell and the electrolyzer. From the results obtained from the experimental setup, the number of start-ups is the same for all the controllers. However, it is a major factor that could reduce the lifespan of the hydrogen path.

Finally, Table 5 shows the range of values of each variable obtained during the experiments by applying the proposed approaches. As seen, the control actions satisfy the constraints given by (2) and (3).

Table 5: Range of values for the states and control inputs obtained during the test period by applying the proposed stochastic MPC controllers.

\begin{tabular}{cccc}
\hline Variable & MS-MPC & TB-MPC & CC-MPC \\
\hline \hline$S O C(\%)$ & {$[57.61,83.73]$} & {$[57.59,75.30]$} & {$[48.82,84.42]$} \\
$M H L(\%)$ & {$[38.51,50]$} & {$[39.07,50]$} & {$[37.06,50]$} \\
$P_{\mathrm{fc}}(\mathrm{W})$ & {$[100,268.13]$} & {$[100,259.69]$} & {$[100,250.44]$} \\
$P_{\mathrm{ez}}(\mathrm{W})$ & {$[100,432.9]$} & {$[100,202.13]$} & {$[100,584.94]$} \\
$P_{\text {grid }}(\mathrm{W})$ & {$[-529.4,320.3]$} & {$[-705.02,314.0]$} & {$[-312.8,117.5]$} \\
\hline
\end{tabular}

In order to extend the comparative analysis to general results and taking into account that the experimental setup of the plant is limited, the non-linear simulation model developed in [39] is used to compare the controllers in other situations and the same circumstances. This simulation model replicates the main dynamics of the 
real plant with enough accuracy. An additional case study for testing the three stochastic MPC controllers and a PF-MPC controller is introduced to enhance the results and obtain conclusions.

Figure 5 shows the evolution of the signals by applying the three stochastic MPC controllers and a PF-MPC controller for a cloudy day in the simulation model of the HyLab microgrid. All controllers present the same evolution to satisfy the demand. The fuel cells are turned on when the power from the renewable sources is not enough to meet the electric demand. Hence, $S O C$ and $M H L$ decrease gradually to supply power to the load. For this particular day, the microgrid imports energy power from the UPG. Given that the excess of renewable energy production over the demand is not enough, the batteries are charged, and the electrolyzer stays off.

To compare the behavior of these MPC controllers, Table 6 shows the results from aforementioned KPIs. The results obtained from the comparison are similar to the previous experimental case study. As expected, the lowest value of $\mathrm{KPI}_{1}$ is presented by standard MPC controller with perfect information; this value gives a target for the comparison. In this sense, CC-MPC controller results in a lower cumulative cost as well as the computational time compared with MS-MPC and TB-MPC controllers. The electrical demand is satisfied by all controllers. Regarding $\mathrm{KPI}_{3}$, MS-MPC controller uses the hydrogen path longer than the other two approaches. Finally, $\mathrm{KPI}_{6}$ shows very similar values for all controllers, the battery $S O C$ is reduced until its lower constrained level. The lowest value of $\mathrm{KPI}_{7}$ is presented by CC-MPC controller because this controller delivers a bigger amount of energy from the fuel cell. Finally, the electrolyzer stays off over the simulation period; therefore $\mathrm{KPI}_{5}$ is zero for all controllers.

Table 6: Comparison of the MS-MPC, TB-MPC, CC-MPC, and PF-MPC controllers applied to the simulation model of HyLab microgrid for a cloudy day by means of $\mathrm{KPI}_{i}, \quad i=1, \ldots, 7$.

\begin{tabular}{lllllll}
\hline Controller & $\begin{array}{l}\mathbf{K P I}_{1} \\
\text { (cost units) }\end{array}$ & $\begin{array}{l}\mathbf{K P I}_{2} \\
(\mathrm{~s})\end{array}$ & $\begin{array}{l}\mathbf{K P I}_{3} \\
(\%)\end{array}$ & $\begin{array}{l}\mathbf{K P I}_{4} \\
\text { (hours) }\end{array}$ & $\begin{array}{l}\mathbf{K P I}_{6} \\
(\%)\end{array}$ & $\begin{array}{l}\mathbf{K P I}_{7} \\
(\%)\end{array}$ \\
\hline \hline MS-MPC & $6.24 \times 10^{12}$ & 7.76 & 0.10 & 6.00 & 40.56 & 20.43 \\
TB-MPC & $5.33 \times 10^{12}$ & 18.20 & 0.11 & 5.67 & 40.64 & 23.46 \\
CC-MPC & $4.22 \times 10^{12}$ & 1.04 & 0.10 & 5.68 & 40.46 & 14.08 \\
PF-MPC & $4.05 \times 10^{12}$ & 0.98 & 0 & 5.90 & 40.01 & 19.77 \\
\hline
\end{tabular}

Table 7 compares the stochastic MPC controllers regarding energy for a cloudy day via simulation. The CC-MPC controller results in higher energy consumption from the fuel cell. The energy from the renewable sources is not enough at the time to turn on the electrolyzer for storing energy as hydrogen. The batteries are used to provide energy to the load; both, MS-MPC and TB-MPC controllers, show a similar use of the energy of the batteries. A remarkable difference is shown in the energy exchanged with the grid, in this case, the TB-MPC controller presents the highest value.

All in all, Table 8 shows priority factors for each one of the proposed stochastic 

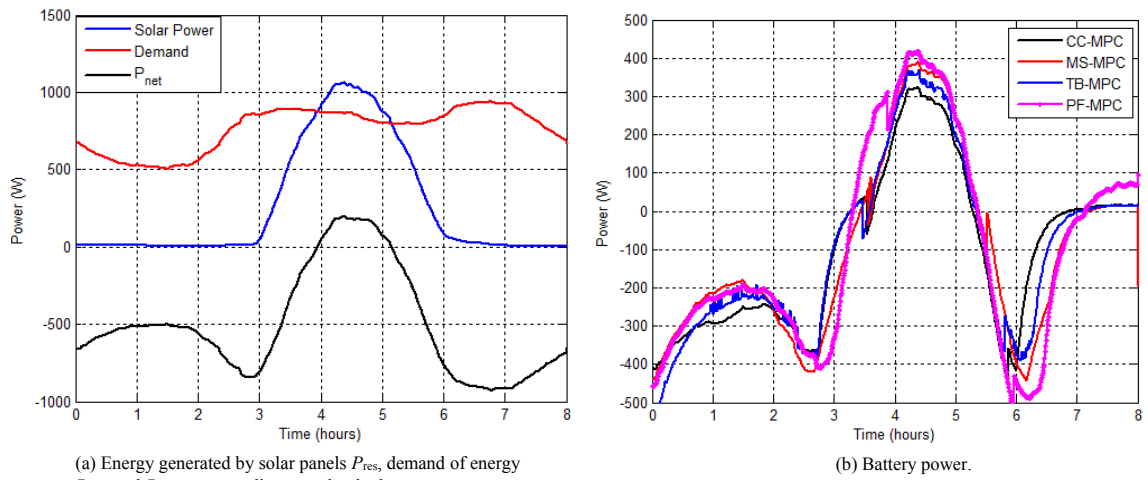

(a) Energy generated by solar panels $P_{\text {res, }}$ demand of energy
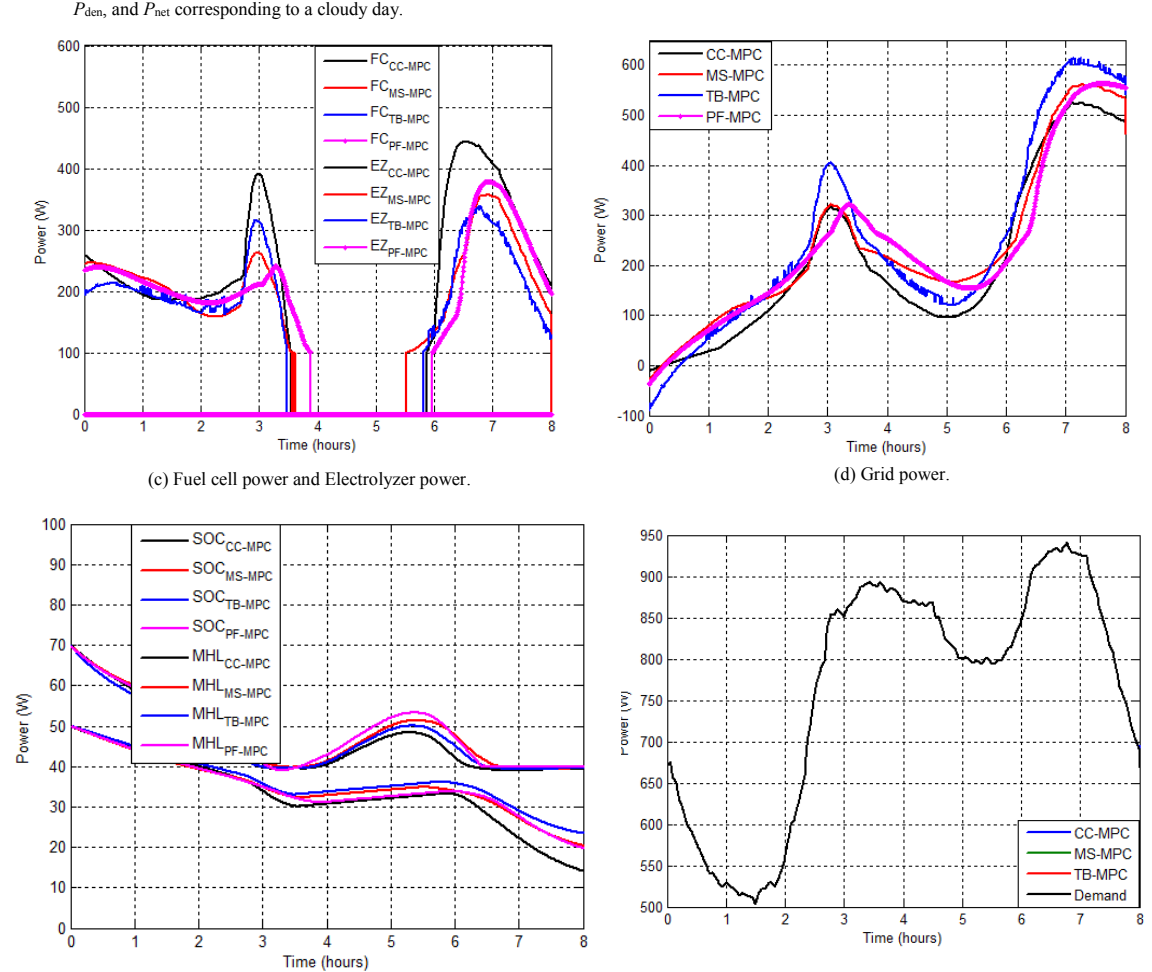

(e) Battery SOC and MHL.

(f) Electric power provided by the microgrid compared with the consumer demand.

Figure 5: Simulation results for a cloudy day applying the proposed stochastic MPC approaches and a standard PF-MPC.

MPC controllers based on the overall analysis at the time of selecting one of them. 
Table 7: Energy produced by the fuel cell, electrolyzer, batteries, and grid during the test period for the simulation model by applying the proposed stochastic MPC controllers.

\begin{tabular}{lllll}
\hline Controller & $\begin{array}{l}E_{\mathrm{fc}} \\
(\mathrm{Wh})\end{array}$ & $\begin{array}{l}E_{\mathrm{ez}} \\
(\mathrm{Wh})\end{array}$ & $\begin{array}{l}E_{\mathrm{batt}} \\
(\mathrm{Wh})\end{array}$ & $\begin{array}{l}E_{\text {grid }} \\
(\mathrm{Wh})\end{array}$ \\
\hline \hline MS-MPC & 472 & 0 & -285 & 688 \\
TB-MPC & 437 & 0 & -287 & 721 \\
CC-MPC & 547 & 0 & -295 & 604 \\
\hline
\end{tabular}

Table 8: Priority factors for selecting one of the proposed stochastic MPC controllers.

\begin{tabular}{|l|c|c|c|}
\hline Priority & MS-MPC & TB-MPC & CC-MPC \\
\hline Maximization of hydrogen path lifespan & & $\checkmark$ & \\
\hline Minimization of energy exchanged with the UPG & & & $\checkmark$ \\
\hline Cumulative cost & & & $\checkmark$ \\
\hline Computational burden & & & $\checkmark$ \\
\hline Demand satisfaction & $\checkmark$ & $\checkmark$ & $\checkmark$ \\
\hline Availability of historical data & $\checkmark$ & $\checkmark$ & \\
\hline
\end{tabular}

\section{Conclusions}

Three stochastic MPC schemes have been designed and applied to a microgrid based on hydrogen storage. Acting on the set-point power of the fuel cell, electrolyzer and grid, the controllers were able to regulate the hydrogen tank level and the state of charge of the battery bank to their desired values. In addition, the controllers consider constraints in both the manipulated variables and the system states for optimal performance and high functionality. Also, the system can deliver electric power from the fuel cell once it has been stored in the form of hydrogen to contribute further to the grid, to satisfy the energy demand under the influence of uncertainties both in the renewal generation and the consumer's behavior.

MS-MPC controller is generally over-conservative because it does not consider the controller capacity to adapt. It calculates a control series valid to all possible scenarios by means an open-loop formulation. However, it is possible to solve the optimization problem by using a control tree and increasing the number of optimization variables and the computational time. Regarding the control point of view, TB-MPC controller works in a closed-loop fashion to adapt the control actions to the expected evolution of the disturbances. Finally, CC-MPC controller formulates the optimization problem by taking into account the statistical features of the uncertainty without increasing the number of variables.

The results obtained with the three presented versions of stochastic MPC controllers show their effectiveness in energy management under economic and optimal criteria. According to the results obtained and their evaluation by the KPIs, it can be said that CC-MPC controller relaxes the constraints of the optimization problem by 
assuming a risk to offer better performance, resulting in a lower cost, less energy exchange with the network when compared to MS-MPC and TB-MPC controllers. This is also the approach with the lowest computational burden. The downside of this approach is that it requires a statical characterization of the disturbances.

The TB-MPC approach, according to the results, provides a more moderate use of the hydrogen path, which could lead to a longer equipment lifespan. From the point of view of the user, the energy demand is fulfilled by increasing energy exchange with the network.

The MS-MPC approach provides a certain robustness of the system, generating control actions able to cope with potential disturbances. This approach provides a trade-off between the time of use of the equipment and the satisfaction of the energy demand.

Other factors that are important to take into account are the initial conditions for $S O C$ and $M H L$. These values will determine the evolution of the variables. Besides, the final value of these variables will take an additional meaning of comparison after a longer time of use of the plant. However, they have been employed in a smaller period to show how they finish after the experiments.

\section{Acknowledgement}

Financial support from the Spanish Ministry of Economy and Competitiveness (COOPERA project, under grant DPI2013-46912-C2-1-R) and the project ECOCIS (Ref. DPI2013-482443-C2-1-R) is acknowledged.

\section{References}

[1] J. P. Lopes, C. Moreira, A. Madureira, Defining control strategies for microgrids islanded operation, IEEE Transactions on Power Systems 21 (2) (2006) 916-924.

[2] L. Valverde, C. Bordons, F. Rosa, Integration of fuel cell technologies in renewable-energy-based microgrids optimizing operational costs and durability, IEEE Transactions on Industrial Electronics 63 (1) (2016) 167-177.

[3] C. Kunusch, C. Ocampo-Martinez, M. Valla, Modeling, diagnosis, and control of fuel-cell-based technologies and their integration in smart grids and automotive systems, IEEE Transactions on Industrial Electronics 62 (8) (2015) 5143-5145.

[4] L. Valverde, F. Rosa, C. Bordons, Design, planning and management of a hydrogen-based microgrid, IEEE Transactions on Industrial Informatics 9 (3) (2013) 1398-1404.

[5] D. Recio, C. Ocampo-Martinez, M. Serra, Design of linear predictive controllers applied to ethanol steam reformers for hydrogen production, International Journal of Hydrogen Energy 37(15) (2012) 11141-11156.

[6] T. Dragicevic, J. Guerrero, J. Vasquez, A distributed control strategy for coordination of an autonomous LVDC microgrid based on power-line signaling, IEEE Transactions on Industrial Electronics 61 (7) (2014) 3313-3326. 
[7] E. F. Camacho, C. Bordons, Model Predictive Control. Second Edition, SpringerVerlag, London, England, 2004.

[8] F. Garcia-Torres, C. Bordons, Optimal economical schedule of hydrogen-based microgrids with hybrid storage using model predictive control, IEEE Transactions on Industrial Electronics 62 (8) (2015) 5195-5207.

[9] M. Pereira, D. Limón, D. Muñoz de la Peña, L. Valverde, T. Alamo, Periodic economic control of a nonisolated microgrid, IEEE Transactions on Industrial Electronics 62 (8) (2015) 5247-5255.

[10] G. Bruni, S. Cordiner, V. Mulone, V. Rocco, F. Spagnolo, A study on the energy management in domestic micro-grids based on model predictive control strategies, Energy Conversion and Management 102 (2015) 50-58.

[11] A. Parisio, E. Rikos, G. Tzamalis, L. Glielmo, Use of model predictive control for experimental microgrid optimization, Applied Energy 115 (2014) 37-46.

[12] J. Patiño, A. Marquez, J. Espinosa, An economic MPC approach for a microgrid energy management system, in: Proceedings of the Transmission Distribution Conference and Exposition - Latin America (PES T D-LA), IEEE PES, Medellín, Colombia, 2014, pp. 1-6.

[13] P. O. Kriett, M. Salani, Optimal control of a residential microgrid, Energy 42 (1) (2012) 321-330.

[14] D. E. Olivares, A. Mehrizi-Sani, A. H. Etemadi, C. A. Cañizares, R. Iravani, M. Kazerani, A. H. Hajimiragha, O. Gomis-Bellmunt, M. Saeedifard, R. PalmaBehnke, G. Jimenez-Estevez, N. Hatziargyriou, Trends in microgrid control, IEEE Transactions on Smart Grid 5 (4) (2014) 1905-1919.

[15] L. I. Minchala-Avila, L. E. Garza-Castañón, A. Vargas-Martínez, Y. Zhang, A review of optimal control techniques applied to the energy management and control of microgrids, Procedia Computer Science 52 (2015) 780 - 787.

[16] D. Bernardini, A. Bemporad, Scenario-based model predictive control of stochastic constrained linear systems, Joint 48th IEEE Conference on Decision and Control and 28th Chinese Control Conference, Shanghai, P.R. China (2009) 63336338.

[17] C. A. Hans, V. Nenchev, J. Raisch, C. Reincke-Collon, A. Younicos, Min-max model predictive operation control of microgrids, in: Proceedings of the 19th IFAC World Congress, Cape Town, South Africa, 2014, pp. 10287-10292.

[18] D. Muñoz de la Peña, A. Bemporad, T. Alamo, Stochastic programming applied to model predictive control, in: Proceedings of the 44th IEEE Conference on Decision and Control, and European Control Conference (CDC-ECC), Seville, Spain, 2005, pp. 1361-1366. 
[19] P. J. van Overloop, S. Weijs, S. Dijkstra, Multiple model predictive control on a drainage canal system, Control Engineering Practice 16 (5) (2008) 531-540.

[20] D. E. Olivares, J. D. Lara, C. A. Cañizares, M. Kazerani, Stochastic-predictive energy management system for isolated microgrids, IEEE Transactions on Smart Grid 6 (6) (2015) 2681-2693.

[21] T. Niknam, R. Azizipanah-Abarghooee, M. R. Narimani, An efficient scenariobased stochastic programming framework for multi-objective optimal micro-grid operation, Applied Energy 99 (2012) 455 - 470.

[22] G. Calafiore, M. Campi, The scenario approach to robust control design, IEEE Transactions on Automatic Control 51 (5) (2006) 742-753.

[23] S. Lucia, T. Finkler, D. Basak, S. Engell, A new robust NMPC scheme and its application to a semi-batch reactor example., In Proc. of the International Symposium on Advanced Control of Chemical Processes, Singapore (2012) 69-74.

[24] M. Petrollese, L. Valverde, D. Cocco, G. Cau, J. Guerra, Real-time integration of optimal generation scheduling with mpc for the energy management of a renewable hydrogen-based microgrid, Applied Energy 166 (2016) 96-106.

[25] J. M. Maestre, L. Raso, P. J. Van Overloop, B. De Schutter, Distributed treebased model predictive control on an open water system, in: Proceedings of the American Control Conference (ACC), Montréal, Canada, 2012, pp. 1985-1990.

[26] Q. Wang, Y. Guan, J. Wang, A chance-constrained two-stage stochastic program for unit commitment with uncertain wind power output, IEEE Transactions on Power Systems 27 (1) (2012) 206-215.

[27] M. Ono, U. Topcu, M. Yo, S. Adachi, Risk-limiting power grid control with an arma-based prediction model, in: Proceedings of the 52nd IEEE Annual Conference on Decision and Control (CDC), Florence, Italy, 2013, pp. 4949-4956.

[28] J. Grosso, C. Ocampo-Martinez, V. Puig, B. Joseph, Chance-constrained model predictive control for drinking water networks, Journal of Process Control 24 (5) (2014) 504-516.

[29] J. M. Grosso, P. Velarde, C. Ocampo-Martinez, J. M. Maestre, V. Puig, Stochastic model predictive control approaches applied to drinking water networks, Optimal Control Applications and Methods In Press. doi:10.1002/oca.2269

[30] A. Hooshmand, B. Asghari, R. Sharma, A novel cost-aware multi-objective energy management method for microgrids, in: Proceedings of the Innovative Smart Grid Technologies (ISGT), IEEE PES, Washington, DC, USA, 2013, pp. 1-6.

[31] Z. Yu, L. McLaughlin, L. Jia, M. C. Murphy-Hoye, A. Pratt, L. Tong, Modeling and stochastic control for home energy management, in: Proceedings of the IEEE Power and Energy Society General Meeting, San Diego, California, USA, 2012, pp. 1-9. 
[32] P. Meibom, R. Barth, B. Hasche, H. Brand, C. Weber, M. O'Malley, Stochastic optimization model to study the operational impacts of high wind penetrations in Ireland, IEEE Transactions on Power Systems 26 (3) (2011) 1367-1379.

[33] T. Hovgaard, L. Larsen, J. Jorgensen, Robust economic MPC for a power management scenario with uncertainties, in: Proceedings of the 50th IEEE Conference on Decision and Control and European Control Conference (CDC-ECC), Orlando, Florida, 2011, pp. 1515-1520.

[34] L. Valverde, F. Rosa, A. del Real, A. Arce, C. Bordons, Modeling, simulation and experimental set-up of a renewable hydrogen-based domestic microgrid, International Journal of Hydrogen Energy 38 (2013) 11672-11684.

[35] C. Bordons, F. García-Torres, L. Valverde, Optimal energy management for renewable energy microgrids (in Spanish), Revista Iberoamericana de Automática e Informática Industrial RIAI 12 (2) (2015) 117-132.

[36] B. S. Lee, H. Y. Park, I. Choi, M. K. Cho, H. J. Kim, S. J. Yoo, D. Henkensmeier, J. Y. Kim, S. W. Nam, S. Park, et al., Polarization characteristics of a low catalyst loading pem water electrolyzer operating at elevated temperature, Journal of Power Sources 309 (2016) 127-134.

[37] A. J. Del Real, A. Arce, C. Bordons, Development and experimental validation of a pem fuel cell dynamic model, Journal of Power Sources 173 (1) (2007) 310324.

[38] M. Tanrioven, M. Alam, Reliability modeling and assessment of grid-connected pem fuel cell power plants, Journal of Power Sources 142 (1) (2005) 264-278.

[39] L. Valverde, F. Rosa, A. del Real, A. Arce, C. Bordons, Modeling, simulation and experimental set-up of a renewable hydrogen-based domestic microgrid, International Journal of Hydrogen Energy 38 (27) (2013) 11672-11684.

[40] G. Schildbach, L. Fagiano, C. Frei, M. Morari, The scenario approach for stochastic model predictive control with bounds on closed-loop constraint violations, Automatica 50 (12) (2014) 3009-3018.

[41] L. Giulioni, Stochastic model predictive control with application to distributed control systems, Ph.D. thesis, Politecnico di Milano (2015).

[42] L. Raso, N. Giesen, P. Stive, D. Schwanenberg, P. Overloop, Tree structure generation from ensemble forecasts for real time control, Hydrological Processes 27 (1) (2013) 75-82.

[43] L. Raso, D. Schwanenberg, N. van de Giesen, P. van Overloop, Short-term optimal operation of water systems using ensemble forecasts, Advances in Water Resources 71 (2014) 200 - 208.

[44] A. Brooke, D. Kendrick, A. Meeraus, R. Raman, General algebraic modeling system (GAMS): A users guide, Boyd \& Fraser publishing company, Danvers, Massachusetts. 
[45] E. Karfopoulos, L. Tena, A. Torres, P. Salas, J. G. Jorda, A. Dimeas, N. Hatziargyriou, A multi-agent system providing demand response services from residential consumers, Electric Power Systems Research 120 (2015) 163 - 176. 